\title{
Terahertz radiation from laser accelerated electron bunches ${ }^{a)}$
}

\author{
W. P. Leemans, ${ }^{\text {b) }}$ J. van Tilborg, ${ }^{\text {c) }}$ J. Faure, ${ }^{\text {d) }}$ C. G. R. Geddes, ${ }^{e)}$ Cs. Tóth, \\ C. B. Schroeder, E. Esarey, and G. Fubianif) \\ Lawrence Berkeley National Laboratory, University of California, Berkeley, California 94720 \\ G. Dugan \\ Cornell University, Ithaca, New York 14853
}

(Received 3 November 2003; accepted 8 January 2004)

\begin{abstract}
Coherent terahertz and millimeter wave radiation from laser accelerated electron bunches has been measured. The bunches were produced by tightly focusing (spot diameter $\approx 6 \mu \mathrm{m}$ ) a high peak power (up to $10 \mathrm{TW}$ ), ultra-short ( $\geqslant 50 \mathrm{fs}$ ) laser pulse from a high repetition rate $(10 \mathrm{~Hz})$ laser system $(0.8 \mu \mathrm{m})$, onto a high density $\left(>10^{19} \mathrm{~cm}^{-3}\right)$ pulsed gas jet of length $\approx 1.5 \mathrm{~mm}$. As the electrons exit the plasma, coherent transition radiation is generated at the plasma-vacuum boundary for wavelengths long compared to the bunch length. Radiation in the $0.3-19 \mathrm{THz}$ range and at 94 $\mathrm{GHz}$ has been measured and found to depend quadratically on the bunch charge. The measured radiated energy for two different collection angles is in good agreement with theory. Modeling indicates that optimization of this table-top source could provide more than $100 \mu \mathrm{J} /$ pulse. Together with intrinsic synchronization to the laser pulse, this will enable numerous applications requiring intense terahertz radiation. This radiation can also be used as a powerful tool for measuring the properties of laser accelerated bunches at the exit of the plasma accelerator. Preliminary spectral measurements indicates that bunches as short as 30-50 fs have been produced in these laser driven accelerators. () 2004 American Institute of Physics. [DOI: 10.1063/1.1652834]
\end{abstract}

\section{INTRODUCTION}

Laser-driven, plasma-based accelerators ${ }^{1}$ hold the potential of becoming compact alternatives to conventional radiofrequency-based linear accelerators (linacs). Accelerating gradients in the $10-100 \mathrm{GV} / \mathrm{m}$ have been measured in laser wakefield accelerator (LWFA) experiments, ${ }^{2-8}$ which is three orders of magnitude higher than in conventional linacs. Acceleration of electron bunches containing several $\mathrm{nC}$ of charge and energy spectra characterized by a Boltzmann-type distribution with an equivalent temperature ranging from the few $\mathrm{MeV}$ to tens of $\mathrm{MeV}$ has been demonstrated in the selfmodulated regime of the LWFA. ${ }^{4-6}$ Recently, experiments have measured electrons with energies above $200 \mathrm{MeV}^{8,9}$ Although the use of laser-plasma accelerators for high energy physics applications will require performance well beyond todays achievements, several applications with less stringent beam property requirements are becoming possible. One of these is radio-isotope production through $(\gamma, n)$ reactions with laser accelerated bunches, ${ }^{10,11}$ which requires a sufficient number of electrons with energy in excess of 10's of $\mathrm{MeV}$, a condition that is relatively straightforward to obtain using laser-plasma-based accelerators. More recently, another application has been explored which exploits the short pulse nature and high charge of the accelerated

\footnotetext{
a) Paper LT 2 2, Bull. Am. Phys. Soc. 48, 200 (2003).

b) Invited speaker.

c) Also at: Technische Universiteit Eindhoven, the Netherlands.

${ }^{d)}$ Presently at Laboratoire d'Optique Appliquée, France.

e) Also at: University of California, Berkeley, USA.

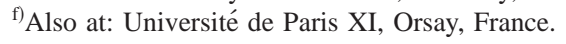

bunches ${ }^{12}$ for the production of coherent radiation. This application will be the focus of the present paper.

The generation of coherent radiation from this electron beam-based radiation source relies on two novel components: (1) Extremely dense, sub-ps electron bunches produced with a compact laser-plasma accelerator and (2) the production of coherent transition radiation by these bunches at the boundary between a plasma and vacuum. ${ }^{12,13}$ This source has the potential for generating up to tens of $\mathrm{mJ}$ per pulse, several orders of magnitude beyond that of conventional $\mathrm{THz}$ radiation sources. Laser-triggered solid-state based sources of $\mathrm{THz}$ radiation have been developed that rely on switched photoconducting antennas (e.g., Ref. 14, and references therein) or optical rectification of femtosecond pulse trains. ${ }^{15}$ Large aperture biased GaAs structures, operated at $1 \mathrm{kHz}$ repetition rate, have produced on the order of $0.5 \mu \mathrm{J} /$ pulse. $^{16}$ Most other sources using either laser switched structures or optical rectification have operated at high frequency (10's of $\mathrm{MHz}$ ) with $\mu \mathrm{W}-\mathrm{mW}$ level average power. The experiments reported in this paper have measured coherent radiation with energies near the $100 \mathrm{~nJ} /$ pulse level, in which the measured energy was limited primarily by the small collection angle $(\sim 100 \mathrm{mrad})$ and the small transverse size $(\sim 100 \mu \mathrm{m})$ of the plasma.

The theory of generation of transition radiation from a relativistic electron bunch crossing a plasma-vacuum boundary will be summarized in Sec. II. Section III discusses the experimental setup and presents evidence for the coherent nature of the radiation at a frequency of $94 \mathrm{GHz}$ and in the $0.3-19 \mathrm{THz}$ range, polarization measurements, and preliminary frequency spectrum measurements. Using these pre- 
liminary spectral measurements, bunch durations on the order of 30-50 fs are inferred. Also compared are the measured and calculated total radiated energy. Section IV summarizes the results presented in this paper and discusses ways of increasing the radiated energy beyond what has been achieved in the present experiments.

\section{THEORETICAL MODEL}

Modeling of the radiation process can be done by assuming that the plasma (with dielectric constant $\epsilon=1-\omega_{p}^{2} / \omega^{2}$, where $\omega_{p}$ and $\omega$ are the plasma and radiation frequencies, respectively) is equivalent to a conductor with a sharp conductor-vacuum boundary. The transition radiation will be generated by electron beam induced polarization currents at plasma densities below the critical density (where $\omega_{p}=\omega$ ) for the radiation wavelength. The plasma density profile used in the experiments discussed in Sec. III had a sufficiently large gradient such that the dielectric constant satisfied $|\epsilon|$ $\gg 1$ within a distance on the order of a skin depth, and therefore, the plasma can be well-modeled as a conductor for frequencies $\omega<\omega_{p}$. In addition, provided the plasma scale length is short compared to the radiation formation length, then the dielectric interface radiates as if it were a sharp dielectric-vacuum boundary.

\section{A. Ideal, monoenergetic bunch}

The energy radiated, per unit frequency $d \omega$ and per unit solid angle $d \Omega$, from a single electron traversing the dielectric boundary is given by the well-known result ${ }^{17-19}$

$$
\frac{d^{2} W_{e}}{d \omega d \Omega}=\left(\frac{r_{e} m_{e} c}{\pi^{2}}\right) \frac{u^{2}\left(1+u^{2}\right) \sin ^{2} \theta}{\left(1+u^{2} \sin ^{2} \theta\right)^{2}}
$$

where $\theta$ is the observation angle with respect to the electron trajectory (assumed to be normal to the plasma surface and along the $z$ axis), $u=\gamma v / c$ is the electron momentum normalized to $m_{e} c, c$ is the speed of light, $m_{e}$ is the electron rest mass, and $r_{e}=e^{2} / m_{e} c^{2}$ is the classical electron radius. The radiation pattern is zero along the axis $(\theta=0)$ and peaks at a radiation cone angle of $\theta \sim 1 / \gamma$ (assuming $\gamma \gg 1$ ). The differential energy radiated by a single electron $d^{2} W_{e} / d \omega d \Omega$ is independent of frequency. In practice, however, the maximum wavelength radiated will be limited, for example, by the physical dimensions of the system, as will be discussed below.

Integrating over solid angle $d \Omega=2 \pi \sin \theta d \theta$ yields

$$
\frac{d W_{e}}{d \omega}=\left(\frac{2 r_{e} m_{e} c}{\pi}\right)\left[\frac{\left(1+2 u^{2}\right)}{u\left(1+u^{2}\right)^{1 / 2}} \tanh ^{-1}\left(\frac{u}{\sqrt{1+u^{2}}}\right)-1\right],
$$

which, in the highly relativistic limit $\gamma \gg 1$, reduces to $d W_{e} / d \omega \simeq(2 / \pi) r_{e} m_{e} c \ln (\gamma)$. The radiation from the beam of electrons sums incoherently for wavelengths short compared to the bunch length, i.e., $W_{\text {ITR }} \simeq N W_{e}$, where $N$ is the number of electrons in the bunch, and a monoenergetic divergenceless beam was assumed. For wavelengths long compared to the bunch length, the radiation sums coherently, i.e., $W_{\mathrm{CTR}} \simeq N^{2} W_{e}$. In particular, the total coherent radiated energy over all angles and frequencies is given by $W_{\text {tot }}$ $\simeq\left(4 r_{e} m_{e} c^{2}\right) N^{2} \ln (\gamma) / \lambda_{\min }$, assuming $\gamma \gg 1$, where $\lambda_{\text {min }}$ is the minimum wavelength for which the bunch radiates coherently and is determined by the electron bunch dimensions, as will be discussed below. The total coherent energy can be written in practical units as

$$
W_{\text {tot }}[\mathrm{J}] \simeq 3.6 \times 10^{-2}(Q[\mathrm{nC}])^{2} \ln (\gamma) / \lambda_{\min }[\mu \mathrm{m}],
$$

where $Q$ is the bunch charge. For example, $\mathrm{Q}=5 \mathrm{nC}(N$ $\left.=3.1 \times 10^{10}\right), \gamma=10$, and $\lambda_{\min }=200 \mu \mathrm{m}$ give $W_{\text {tot }} \simeq 10 \mathrm{~mJ}$, which is several orders of magnitude beyond that of conventional sources.

\section{B. Energy spread and divergence effects}

The above analysis can be generalized for the case of an arbitrary electron beam momentum distribution. Assuming full coherence, and for the case of a divergenceless beam with normalized energy distribution $g(u)$, the differential energy spectrum of the coherent transition radiation is

$$
\begin{aligned}
\frac{d^{2} W_{\mathrm{CTR}}}{d \omega d \Omega}= & \left(\frac{r_{e} m_{e} c}{\pi^{2}}\right) N(N-1) \\
& \times \sin ^{2} \theta\left|\int \operatorname{dug}(u) \frac{u\left(1+u^{2}\right)^{1 / 2}}{1+u^{2} \sin ^{2} \theta}\right|^{2} .
\end{aligned}
$$

In general, the differential energy spectrum Eq. (4) will be modified by finite beam divergence. For fully coherent radiation, the energy emitted by a cylindrically symmetric beam distribution with small divergence angle $\psi$ and a small observation angle $\theta$, such that $u^{2} \psi^{2}<u^{2} \theta^{2}<1$, is ${ }^{13}$

$$
\begin{aligned}
\frac{d^{2} W_{\mathrm{CTR}}}{d \omega d \Omega} \simeq\left(\frac{r_{e} m_{e} c}{\pi^{2}}\right) N(N-1) \frac{\theta^{2}}{4}\left[\left(1+2\left\langle u^{2}\right\rangle\right)^{2}\right. \\
\left.-2\left(1+2\left\langle u^{2}\right\rangle\right)\left(3\left\langle u^{2}\right\rangle+4\left\langle u^{4}\right\rangle\right)\left\langle\psi^{2}\right\rangle\right],
\end{aligned}
$$

for $u^{2}>1$, where $\left\langle\psi^{2}\right\rangle^{1 / 2}$ is the root-mean square (rms) divergence of the electron beam through the plasma-vacuum boundary and the brackets indicate an average over the momentum distribution. For $\theta^{2} \ll \psi^{2}<1, d^{2} W_{\mathrm{CTR}} / d \omega d \Omega \simeq 0$, and no coherent radiation is observed on axis for a cylindrically symmetric beam. Physically, this result is due to the fact that the radiation fields produced by the electrons are summed for coherent radiation and the cylindrical symmetry results in the sum vanishing on axis, whereas, in the case of incoherent radiation, the intensities radiated by each electron are summed, resulting in a nonzero incoherent radiation intensity on axis for a beam with finite divergence. As Eq. (5) indicates, the effect of beam divergence is to reduce the coherent transition radiation energy.

Using Eq. (5), the total coherent radiation energy into a small collection angle $\theta \leqslant \theta_{\text {coll }}<1 /\langle u\rangle$, and for a bandwidth $\Delta \omega / \omega$ is

$$
W_{\mathrm{CTR}} \simeq m_{e} c^{2}\left(r_{e} / \lambda\right) N(N-1)(\Delta \omega / \omega) \theta_{\mathrm{coll}}^{4}\left\langle u^{2}\right\rangle^{2},
$$

assuming $\left\langle u^{2}\right\rangle>1$ and $\left\langle\psi^{2}\right\rangle \ll 1 /\left\langle u^{2}\right\rangle$. Laser-plasma generated electron beams in the self-modulated regime can be characterized by a Boltzmann momentum distribution $g(u)$ $=u_{\mathrm{T}}^{-1} \exp \left[-u / u_{\mathrm{T}}\right]$, where $u_{\mathrm{T}}$ is the temperature of the distribution. The amount of radiated energy in the collection cone 
half-angle $\theta_{\text {coll }}<1 / u_{\mathrm{T}}<1$ and frequency bandwidth $\Delta \omega / \omega$ is given by $\Delta W_{e} \simeq 4 m_{e} c^{2}\left(r_{e} / \lambda\right) N^{2}\left(u_{\mathrm{T}} \theta_{\text {coll }}\right)^{4}(\Delta \omega / \omega)$. For example, $\Delta W_{e}=30 \mu \mathrm{J}$ within $\theta_{\text {coll }}=50 \mathrm{mrad}$ for a $1.5 \mathrm{nC}$ bunch with temperature $u_{\mathrm{T}}=9$ in a bandwidth $0.3-3 \mathrm{THz}$. Due to the strong dependence on electron energy and angle, $W_{\mathrm{CTR}} \propto\left(\gamma \theta_{\text {coll }}\right),{ }^{4}$ the measured energy can easily be increased by increasing either the electron energy $\gamma$ or the cone angle $\theta_{\text {coll }}$ of the collection optics (for $\theta_{\text {coll }}<1 /\langle u\rangle$ ).

\section{Finite bunch size and plasma radius effects}

The spectrum of the radiation and total energy radiated will be strongly influenced by the effects of coherence and the transverse size of the dielectric structure (diffraction from the plasma edge). Including the effects of coherence and diffraction from the transverse edge of the plasma, the differential radiated energy by a bunch traveling normal to the plasma-vacuum boundary is ${ }^{13}$

$$
\begin{aligned}
\frac{d^{2} W_{e}}{d \omega d \Omega}= & \frac{r_{e} m_{e} c}{\pi^{2}} N(N-1) \sin ^{2} \theta \mid \int d u g(u) F(\omega, \theta, u) \\
& \times\left.\frac{u\left(1+u^{2}\right)^{1 / 2}}{\left(1+u^{2} \sin ^{2} \theta\right)} D(\omega, \rho, \theta, u)\right|^{2},
\end{aligned}
$$

where

$$
F=\int d z d^{2} \vec{r}_{\perp} e^{-i \vec{k}_{\perp} \cdot \vec{r}_{\perp}} e^{-i \omega z / v} f\left(\vec{r}_{\perp}, z\right),
$$

is the Fourier transform of the electron beam spatial distribution $f\left(\vec{r}_{\perp}, z\right)$, or spatial form factor, $\vec{k}$ is the radiation wave vector, and

$$
\begin{aligned}
D= & 1-J_{0}(b u \sin \theta)\left[b K_{1}(b)+b^{2} K_{0}(b) / 2\right] \\
& -b^{2} K_{0}(b) J_{2}(b u \sin \theta) / 2,
\end{aligned}
$$

is the correction due to diffraction from the transverse edge of the plasma. Note that Eq. (8) assumes that there are no correlations between position and momentum. The parameter $b \equiv k \rho / u$ describes the relative influence of the transverse boundary (i.e., the ratio of the transverse size of the plasma $\rho$ to the transverse extent of the self-fields of the relativistic electrons $\sim \gamma \lambda$ ), and $K_{0}, K_{1}, J_{0}$, and $J_{2}$ are Bessel functions. For large transverse size (i.e., $b \gg 1$ ), $D \simeq 1$, and the coherent radiation is well-described by transition radiation. For $b \sim 1$, (i.e., transverse plasma size small compared to the effective source size $\gamma \lambda$ ) diffraction effects will strongly modify the radiated energy spectrum.

The degree of coherence in the bunch will be determined by the form factor Eq. (8). For a bunch with an uncorrelated Gaussian spatial distribution, the spatial form factor is $F=F_{\perp} F_{\|}, \quad$ where $\quad F_{\perp}=\exp \left[-\left(k \sigma_{\perp} \sin \theta\right)^{2} / 2\right]$ and $F_{\|}$ $=\exp \left[-\left(\omega \sigma_{\|} / v\right)^{2} / 2\right]$, with $\sigma_{\perp}$ the rms transverse bunch radius and $\sigma_{\|}$the rms longitudinal bunch length. Fully coherent emission in both the transverse and longitudinal directions requires $F \simeq 1$. This condition is satisfied when $\sigma_{\perp} \sin \theta$ and $\sigma_{\|}$are much less than the radiation wavelength. Measurement of the energy spectra provides a method for determining the spatial distribution of the electron beam.
As Eq. (7) indicates, the finite transverse extent of the plasma produces a wavelength dependence in the differential energy for fully coherent radiation. The spectral content of the radiation is no longer constant, as is the case for transition radiation from a fully coherent beam traversing a boundary of infinite transverse extent, and the spectra is strongly modified by the diffraction effects for parameters $b \sim 1$ (i.e., $\lambda \sim \rho / \gamma)$. Distortion of the spectra increases with larger energy, and the spectra are suppressed for decreasing transverse size. For large transverse size $(b \gg 1)$, the fully coherent spectra becomes constant (i.e., the limit of transition radiation from an infinite transverse boundary). In general, the spectral region of coherent radiation is approximately $2 \pi \sigma_{z}<\lambda<2 \pi \rho / u$, where the lower bound is due to longitudinal coherence [ $F$ in Eq. (7)], and the upper bound is due to diffraction effects $[D$ in Eq. (7)].

\section{EXPERIMENTAL SETUP AND MEASUREMENTS}

\section{A. Setup}

The experiments described in this paper used the short pulse, high peak power and high repetition rate Ti: $\mathrm{Al}_{2} \mathrm{O}_{3}$ laser system ${ }^{10}$ of the l'OASIS facility at Lawrence Berkeley National Laboratory. Low energy laser pulses (of wavelength $\lambda \simeq 0.8 \mu \mathrm{m}$ ) from a Ti: $\mathrm{Al}_{2} \mathrm{O}_{3}$ laser oscillator were first temporally stretched, amplified to $1 \mathrm{~J} /$ pulse level, and then compressed using a grating based optical compressor. Following compression, the laser beam was focused to a spot size $w$ $=6 \mu \mathrm{m}$ with a $30 \mathrm{~cm}$ focal length $(\mathrm{F} / 4)$ off-axis parabola onto a pulsed gas jet. The peak power $P$ of the laser was varied using both the pulse duration and laser energy. At optimum compression $[55$ fs full-width-half-maximum (FWHM) duration], $P \simeq 8.3 \mathrm{TW}$, resulting in a calculated peak intensity $I=2 P / \pi r_{s}^{2} \simeq 1.5 \times 10^{19} \mathrm{~W} / \mathrm{cm}^{2}$ and a normalized laser strength $a_{0} \simeq 8.6 \times 10^{-10} \lambda[\mu \mathrm{m}] I^{1 / 2}\left[\mathrm{~W} / \mathrm{cm}^{2}\right]$ $\simeq 2$.6. The gas jet was backed with up to 70 bar helium gas. The profile of the jet had a $1.8 \mathrm{~mm}$ flat top at a density of about $3 \times 10^{19} \mathrm{~cm}^{-3}$ with a ramp of $1 \mathrm{~mm}$ length to zero density on either side (a total length of just under $4 \mathrm{~mm}$ ). The plasma density profile, measured with a folded-wave interferometer using $400 \mathrm{~nm}$ wavelength, 50 fs duration laser pulses, had a typical transverse size of $100 \mu \mathrm{m} \pm 15 \mu \mathrm{m}$. The lay-out of the experiment in shown in Fig. 1.

The charge per bunch and electron beam spatial profile were measured using an integrating current transformer (ICT) and a phosphor screen, located 50 and $75 \mathrm{~cm}$ away from the exit of the gas jet, respectively. The response of the phosphor screen (number of counts on the CCD vs deposited charge) was calibrated against the ICT. The energy distribution was obtained using an imaging magnetic spectrometer ${ }^{20}$ and a phosphor screen with CCD camera. At a given excitation current of the magnet, the momentum acceptance of the magnetic spectrometer was $\delta p / p= \pm 2 \%$. A spectrum ranging from 0 to $50 \mathrm{MeV}$ was obtained by recording the total light yield on the CCD image (corrected for background counts) for excitation currents ranging from 0 to $45 \mathrm{~A}$. 


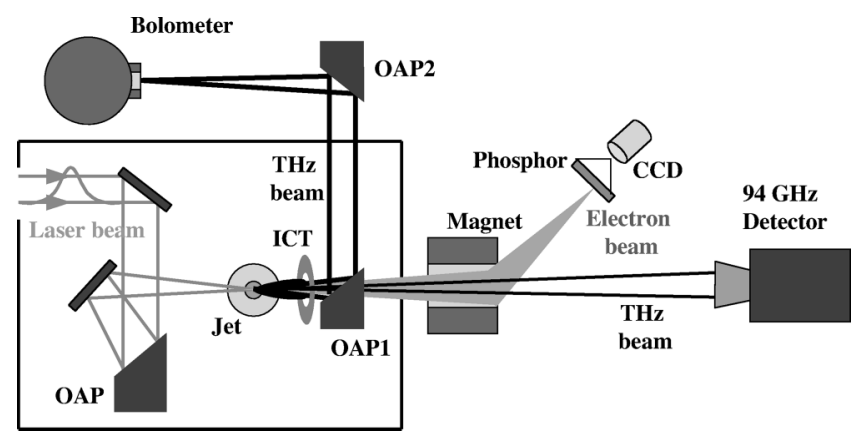

FIG. 1. Schematic of the experimental setup. The high power laser beam is focused using an off-axis parabolic mirror (OAP) onto a high pressure pulsed gas jet operating with about 70 bar He backing pressure. An integrating current transformer (ICT) is used to measure the charge per bunch of the electron beam produced in the laser ionized gas jet. A double focusing magnetic spectrometer is used to measure the electron beam energy by scanning the magnet excitation current. Radiation is collected with the use of a second off-axis parabola (OAP1) with a hole in the middle to transmit the high power laser beam and electron beam. OAP1 collimates the radiation which is transmitted through a silicon window and then focused using OAP2 onto the bolometer. This optical system collects radiation with angular direction between 80 and $300 \mathrm{mrad}$. The measurements of the radiated energy in a $30 \mathrm{mrad}$ solid angle were done by focusing the radiation onto the bolometer using a metal coated spherical mirror with a $50 \mathrm{~cm}$ focal length, located about $1 \mathrm{~m}$ away. The measurements of radiation at $94 \mathrm{GHz}$ used a heterodyne detection system. The radiation exited the vacuum chamber through a 75 micron thick kapton window and was collected with a microwave horn ( $25 \mathrm{mrad}$ collection angle).

\section{B. Electron beam properties}

A typical energy spectrum is shown in Fig. 2 and is reasonably well approximated by a Boltzmann distribution with a temperature of $4.6 \mathrm{MeV}$. The electron beam profile was found to be dependent on laser power. Two representative electron beam images are shown in Fig. 3. The beam divergence vs laser pulse duration is shown in Fig. 4. The laser pulse duration was changed by changing the distance

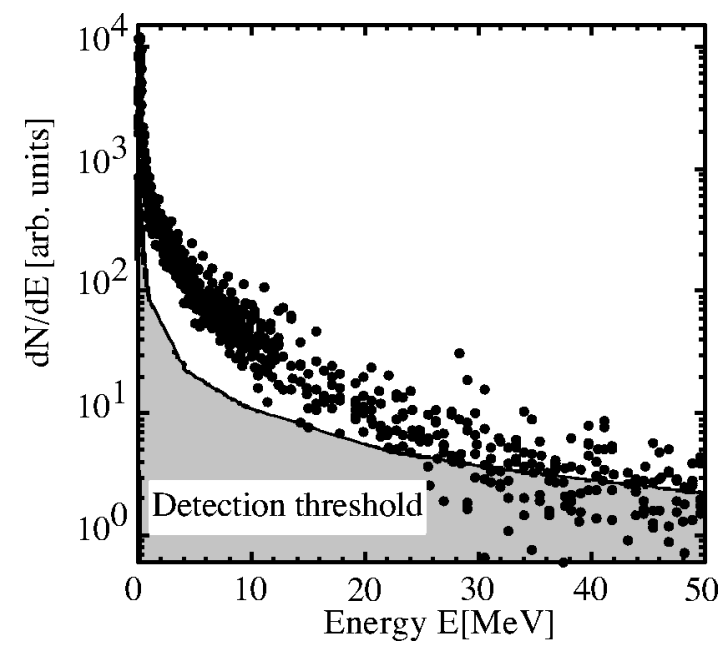

FIG. 2. Electron energy spectrum $d N / d E$ measured using a double focusing imaging magnetic spectrometer. The spectrum was obtained by scanning the excitation current in the magnet and measuring the intensity on a phosphor screen. For any given current, the momentum acceptance $\delta p / p$ of the spectrometer was $\pm 2 \%$. Each data point represents 10 shots. The spectrum is reasonably well approximated by a Boltzmann distribution with an effective temperature of $4.6 \mathrm{MeV}$.

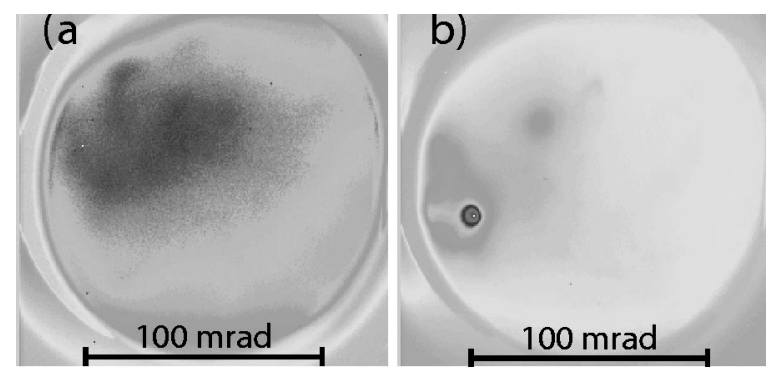

FIG. 3. Electron beam images measured by imaging a $10 \mathrm{~cm}$ diameter phosphor screen located $75 \mathrm{~cm}$ away from the gas jet with a CCD camera, for laser peak power (a) around 2-4 TW and (b) around $8 \mathrm{TW}$. The unnormalized geometric rms "emittance" for the small spot is $0.01 \mathrm{~mm}$-mrad, obtained by multiplying the assumed source size from which the beam originates [ $6 \mu \mathrm{m}$ full width at half maximum (FWHM), i.e., equal to laser spot size] and a $10 \mathrm{mrad}$ (FWHM) divergence angle.

between the gratings in the pulse compressor. The asymmetry in the data curve for points on the left- and on the righthand side of the minimum pulse duration is consistent with previous observations of a dependence of electron yield on the shape of the laser pulse. ${ }^{7,21}$

For power levels near the maximum power, electron beams were observed with a divergence less than $10 \mathrm{mrad}$ (FWHM) [see Fig. 3(b)]. The amount of charge contained in the small beams was typically about $5 \%$ of the main beam and ranged between 30 and $50 \mathrm{pC}$. The energy of this part of the beam was not measured separately. However, the shotto-shot fluctuation in beam divergence near maximum power was found to be significantly larger than for the lower power cases, as can be seen in Fig. 4 from the relatively larger error bars. In addition, the pointing varied from shot-to-shot by more than $20 \mathrm{mrad}$. For power levels which exceed the critical power for self-focusing by a factor of 5 or more, the laser self-channeling and particle trapping processes operate in a highly nonlinear regime, which likely results in a loss of reproducibility due to sensitivity to operating parameters. In addition, pre-pulse effects have been shown to be important in the acceleration process and the production of stable beams. $^{22}$

Assuming that the electron beam originated from a spot size equal to the laser spot size $w$, a value for an unnormal-

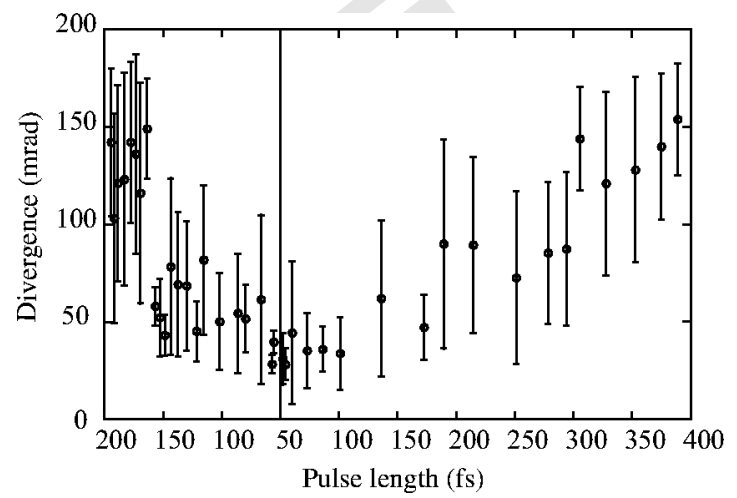

FIG. 4. Electron beam divergence vs laser pulse duration. The laser pulse duration was varied by changing the distance between the gratings in the pulse compressor and the duration was measured using a single-shot second order autocorrelator. Each data point represents 10 shots. 
ized rms geometric "emittance" for the high power case can be estimated to be $0.01 \mathrm{~mm}$ mrad, by simply multiplying the assumed source size and measured divergence angle. Similarly low emittance values between 0.01 and $0.1 \pi \mathrm{mm}$ mrad have been reported previously. ${ }^{22,23}$ However, prior to interpreting this quantity into an electron beam emittance value, as commonly used for conventional accelerators, a thorough understanding of all phase space properties of the beam is required, including energy (mean and spread) and bunch length, as a function of propagation distance. Upon exiting the plasma, the high density electron bunch is subjected to significant space charge forces that can result in transverse beam blow-up as well as modifications to the longitudinal bunch shape and energy distribution. In general, longitudinal space charge forces are expected to lengthen the bunch, thereby reducing the density and, consequently, the effect of transverse space charge forces while the beam is propagating through vacuum. ${ }^{24,25}$ Also, the large energy spread can result in ballistic spreading of the electron bunches since fast electrons would out run the slower ones. More complicated modifications of the energy distribution and correlations in momentum and physical space can still occur however, complicating the interpretation of the data. As an example, if the bunch initially has slow electrons in the front and back, and fast electrons in the middle, bunch compression can occur during the propagation resulting in a temporary increase in space charge forces. Detailed measurements of the energy (mean and spread), bunch length, and transverse characteristics of these low divergence beams are needed to fully understand their behavior during propagation in vacuum and to properly assign an emittance value. Pepper pot based measurements of electrons that were energy filtered around 55 $\mathrm{MeV}$ have recently been carried out in a similar laser wakefield setup to ours and indicate a normalized emittance of $3 \pi \mathrm{mm}$ mrad at the spectrometer location. ${ }^{26}$

\section{Radiation measurements}

The radiation measurements were done in two different frequency ranges: At $94 \mathrm{GHz}$ using a mm-wave heterodyne detection system and from 0.3 to $19 \mathrm{THz}$ using a liquid helium cooled bolometer. Radiation at $3.2 \mathrm{~mm}$ wavelength (94 $\mathrm{GHz}$ ) was measured using a narrowband $(1 \mathrm{GHz})$ microwave heterodyne detector system consisting of two radiofrequency diodes: An Impatt diode operating at $94 \mathrm{GHz}$ and a Gunn diode, which was frequency tuned and locked at a $450 \mathrm{MHz}$ frequency difference (fifth harmonic of the laser oscillator frequency) with the impatt diode. This particular choice of difference frequency provided for proper synchronization between the arrival of the radiation burst, intrinsically synchronized to the laser pulse, and the Gunn diode. Radiation was collected and focused with a $50 \mathrm{~mm}$ diameter polycarbonate lens (focal length $=15 \mathrm{~cm}$ ) into a microwave horn, located $2 \mathrm{~m}$ from the gas jet (full collection angle $=25 \mathrm{mrad}$ ), and downconverted by mixing it with the Gunn diode signal.

To verify the dependence of radiated energy on collection angle, two different setups were used in the higher frequency ranges. In the first setup, with a collection angle of
$30 \mathrm{mrad}$, radiation was reflected out of the vacuum chamber through a window using a $5 \mu \mathrm{m}$ thick metal coated nitrocellulose foil $30 \mathrm{~cm}$ from the gas jet. (Although transition radiation will be generated when the beam propagates through this foil, the emission is incoherent due to bunch lengthening ${ }^{25}$ and transverse beam size increase at that location, measured with a phosphor screen.) The radiation then propagated in air and through laser beam attenuators, and was focused with an F/30 metal coated spherical mirror onto a cooled (4.2 K) Si-based bolometer, equipped with an internal $3 \mathrm{THz}$ low-pass filter, to measure the radiated energy. The spectral transmission properties of all solid materials used in the T-ray path were measured using a ZnTe based optical rectification system. ${ }^{27}$

To improve the collection efficiency, the second setup used an off-axis $90^{\circ}$ parabola (effective focal length of 127 $\mathrm{mm}$ ) to collimate $\mathrm{THz}$ light onto a second off-axis parabola (effective focal length of $177.8 \mathrm{~mm}$ ). After exiting the vacuum chamber through a silicon window, the radiation was incident on the bolometer which was positioned in the calculated focal position. Note that a hole was drilled on axis through the first parabola to allow the $800 \mathrm{~nm}$ laser beam to propagate through the parabola, resulting in a collection angle (half-cone) covering emission angles between $80<\theta$ $<300 \mathrm{mrad}$. The complete bolometer system sensitivity (volts per incident energy) including a gain factor of the internal amplifier of 1000 and the bolometer internal collection efficiency was calibrated and found to be $1 \mathrm{~V}$ of signal per $4.0 \mathrm{pJ}$ of incident THz energy. The total transmission of all materials in the THz beam path was measured by a farinfrared interferometer in combination with a hot $\mathrm{Hg}$ lamp and found to be $2.5 \times 10^{-5}$.

For $\theta_{\text {coll }}=30 \mathrm{mrad}$, and electron beams containing up to $1.5 \mathrm{nC}$, the measured radiated energy per pulse between 0.3 and $2.9 \mathrm{THz}$ was between 3 and $5 \mathrm{~nJ} /$ pulse. $^{12}$ For the setup with collection angle covering 80-300 mrad angles, the total radiated energy in the $\mathrm{THz}$ beam was approximately $70-80$ $\mathrm{nJ}$ for electron bunches containing about $0.3 \mathrm{nC}$.

For the experimental parameters $[\rho=100 \mu \mathrm{m} \pm 15 \mu \mathrm{m}$, $\theta_{\text {coll }}=30 \mathrm{mrad}_{-10 \mathrm{mrad}}^{+0 \mathrm{mrad}}, 1.5 \mathrm{nC}$ charge, and $u_{\mathrm{T}}$ $=9(4.6 \mathrm{MeV})]$ the calculated energy per pulse is $2.3 \mathrm{~nJ}$, which agrees within error bars with the 3-5 $\mathrm{nJ}$ measured experimentally. For the second case, using $0.3 \mathrm{nC}$ charge, frequencies ranging from 0.3 to $19 \mathrm{THz}$, and angular coverage from 80 to $300 \mathrm{mrad}$, the calculated energy per pulse is $160 \mathrm{~nJ}$, compared to $70-80 \mathrm{~nJ}$ experimentally. This is in reasonable agreement, given the sensitivity of the radiated energy to bunch length, plasma size, energy distribution and total charge.

To verify that the radiation is emitted coherently, the bunch charge was varied by changing either the laser pulse duration or the position of the gas jet with respect to the laser beam focal position. ${ }^{10,7}$ The dependence of the peak amplitude of the bolometer signal as well as $94 \mathrm{GHz}$ signal versus bunch charge is shown in Figs. 5 and 6, respectively. Radiation collected on the bolometer scales quadratic with charge consistent with coherent emission of radiation. Radiation levels at $94 \mathrm{GHz}$ scale roughly quadratic as well, although for charge less than $<0.8 \mathrm{nC}$ the scaling was closer to linear, 


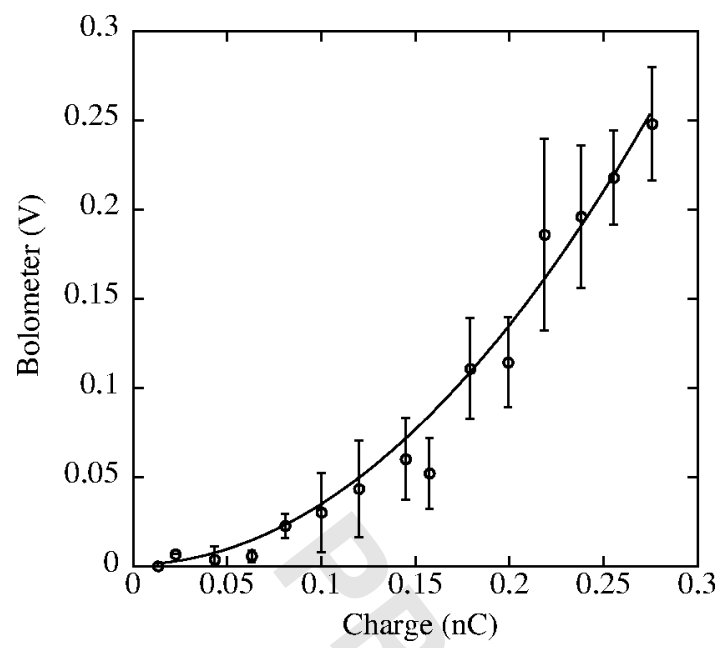

FIG. 5. Bolometer voltage vs charge per bunch for the $80-300 \mathrm{mrad}$ collection half-angle. Each dot represents an average of 50 experimental shots, with rms variation as error bar. The solid line is a quadratic fit to the data, indicating coherence of the radiated signal. The bolometer (Infrared Laboratories) had a voltage responsivity $S=2.73 \times 10^{8} \mathrm{~V} / \mathrm{W}$ and thermal conductivity $G=17.01 \mu \mathrm{W} / \mathrm{K}$. Since the radiation bursts are much shorter than the bolometer time constant, $\tau_{\text {bolo }}=0.33 \mathrm{~ms}$, the detector operates as a calorimeter. The absorbed energy is then given by: $E_{\text {absorbed }}$ $=G \tau_{\text {bolo }} \Delta V_{\text {bolo }} /\left(R \eta_{\text {bolo }}\right)=4.0 \times \Delta V_{\text {bolo }}$ (in pJ), where $R=S \times G=4.64$ is the induced voltage per $\mathrm{K}$ of temperature rise (in $\mathrm{V} / \mathrm{K}$ ), $\Delta V_{\text {bolo }}$ is the height of the voltage pulse (in $\mathrm{V}$ ), and $\eta_{\text {bolo }} \sim 0.3$ is the bolometer efficiency. The radiated energy is $E_{\text {radiated }}=E_{\text {absorbed }} / \eta_{\text {coll }}$, where $\eta_{\text {coll }}$ is the overall collection efficiency of the system.

as can be seen in Fig. 6. The offset in the GHz detector data is due to an intrinsic bias level in the electronics. Radiation for frequencies lower than $0.5 \mathrm{THz}$ (e.g., $94 \mathrm{GHz}$ ) is significantly reduced due to diffraction effects caused by the finite transverse plasma size for this long wavelength radiation. The signal reduction compared to an infinite size plasma scales as $0.5(\pi \rho \theta / \lambda)^{4}$ and, assuming a $100 \mu \mathrm{m}$ radius plasma, a collection angle of $0.025 \mathrm{rad}$, and a $3.2 \mathrm{~mm}$ wavelength, is equal to a factor of $2 \times 10^{-11}$. This is consistent

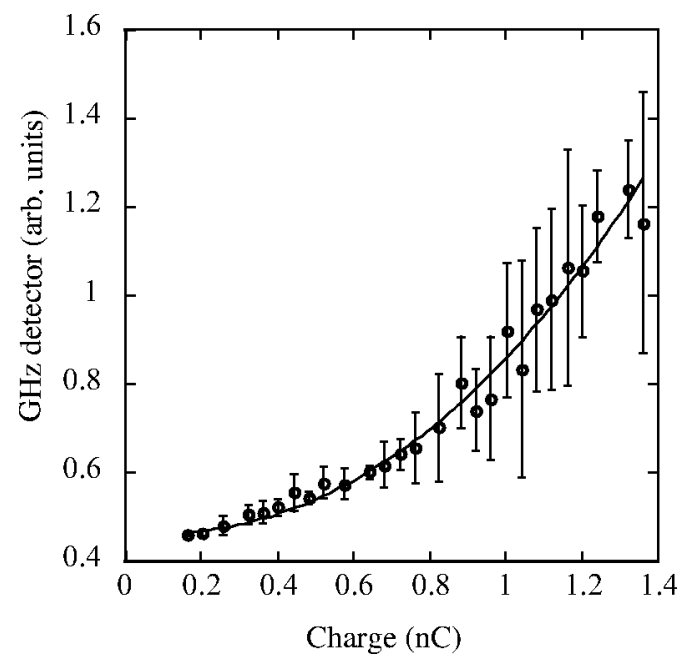

FIG. 6. $94 \mathrm{GHz}$ signal voltage vs charge per bunch. Each dot represents an average of 50 experimental shots, with rms variation as error bar. The solid line is a quadratic fit to the data, indicating coherence of the radiated signal. with the signal still being still than incoherent radiation and background sources and showing the quadratic dependence on charge.

\section{Polarization measurement}

Since transition radiation is in principle radially polarized for an axisymmetric e-beam, a further test of the validity of our model was carried out by measuring the polarization characteristics of the source. A far-infrared wire-grid linear polarizer was placed at the entrance plane of the bolometer, where the $\mathrm{THz}$ radiation is brought to a focus. The polarizer angle was rotated from $0^{\circ}$ to $180^{\circ}$ in steps of $30^{\circ}$ and at each position about 30 shots were acquired. Within the error bars, the transmitted signal was found to be independent of the polarizer angle, consistent with radially polarized radiation.

\section{E. Radiation spectrum}

As a first attempt to obtain spectral information of the $\mathrm{THz}$ source, the total radiated energy was collected in the $0.3-19 \mathrm{THz}$ and in the $0.3-2.9 \mathrm{THz}$ ranges (and hence 2.9-19 THz range). This was done with the use of two separate filters that were inserted between the bolometer window and the bolometer detector element. The first long pass filter consisted of a thin polyethylene filter with a diamond scattering layer, and had a cut-on wavelength of $16 \mu \mathrm{m}(19$ $\mathrm{THz}$ ). The second long pass filter, made of crystal quartz with a broadband anti-reflection coating, had a cut-on wavelength of $103 \mu \mathrm{m}(2.9 \mathrm{THz})$. Radiation at wavelengths longer than $1 \mathrm{~mm}(0.3 \mathrm{THz})$ was not detectable with the bolometer through its intrinsic design.

Analysis of the raw experimental data (averaging of several tens of shots) shows that $22 \%$ of the observed radiation was emitted in the frequency range $0.3-2.9 \mathrm{THz}$, while $78 \%$ of the radiation was emitted within the frequency range 2.9-19 THz range. To model this data, the radiated spectrum was calculated including diffraction (see Sec. II) from a 100 $\mu \mathrm{m}$ radius plasma column and again using a $4.6 \mathrm{MeV}$ Boltzmann distribution for the electron bunch energy distribution. The spectrum of the radiation can be calculated from Eq. ?? assuming a longitudinal charge distribution and transverse size of the dielectric (plasma). Figure 7 shows the calculated spectrum of the radiation for a Gaussian longitudinal charge distribution with rms length $\sigma_{z}=13.5 \mu \mathrm{m}$ and $u_{\mathrm{T}}$ $=9$, transitioning out of a plasma with a transverse halfwidth of $100 \mu \mathrm{m}$. The short wavelength cutoff is due to the longitudinal coherence effects $\left(\lambda_{\min } \sim \sigma_{z}\right)$, and the long wavelength cutoff is due to the influence of diffraction radiation from the transverse edge of the plasma $\left(\lambda_{\max } \sim \rho / u_{\mathrm{T}}\right)$. For these values of plasma size and electron beam properties, the spectrum shown in Fig. 7 contains 22\% of the radiation emitted in the frequency range $0.3-2.9 \mathrm{THz}$, and $78 \%$ of the radiation emitted within the frequency range $2.9-19 \mathrm{THz}$ range. An rms bunch length on the order of $10 \mu \mathrm{m}$ is consistent with self-modulated LWFA simulations ${ }^{28}$ which typically predict electron beam bunch lengths to be on the order of the laser pulse duration. 


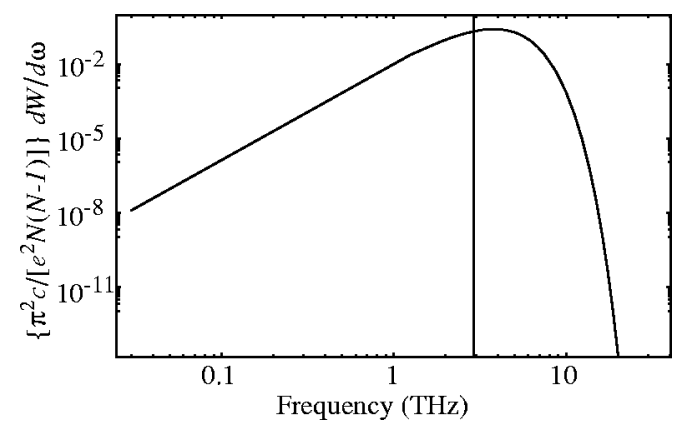

FIG. 7. Calculated radiated energy vs frequency within $80<\theta_{\text {coll }}$ $<300 \mathrm{mrad}$. The measured electron beam energy distribution was approximated using a Boltzmann distribution with a temperature of $4.6 \mathrm{MeV}$, the longitudinal electron beam distribution was assumed to be a Gaussian with $13.5 \mu \mathrm{m}$ root-mean squared (rms) length, and the plasma to have a transverse half-width $=100 \mu \mathrm{m}$. For these parameters, $22 \%$ of the radiated energy is emitted for frequencies $<2.9 \mathrm{THz}$ and $78 \%$ of energy for frequencies $>2.9 \mathrm{THz}$, as measured in the experiment.

\section{SUMMARY AND CONCLUSIONS}

This work has presented theoretical and experimental results on the generation of transition radiation from laser accelerated electron bunches crossing a plasma-vacuum boundary. An LWFA produced fs duration electron bunches containing several $\mathrm{nC}$ of charge. Transition radiation at the plasma boundary occurs while the bunch is still very dense. This radiation is coherent and scales quadratically with charge for wavelengths long compared to the bunch length. The radiation is directed and intrinsically synchronized to the laser beam. The total radiated energy versus bunch charge was experimentally found to scale quadratically, consistent with a coherent emission process. The energy transmitted through a linearly polarizing wire grid was found to be independent of the rotation angle of the polarizer, consistent with radial polarization expected for transition radiation. Preliminary (coarse) spectral measurements were carried out using a set of long pass filters. The experimentally measured fraction of energy in the ranges $0.3-2.9 \mathrm{THz}$ and $2.9-19 \mathrm{THz}$ was $22 \%$ and $78 \%$, respectively. The long and short wavelength spectral cut-off was shown to depend on the transverse plasma profile and bunch length, repectively. Good agreement with theory was obtained by assuming an electron bunch length $\sim 10 \mu \mathrm{m}$ and a plasma radius $\sim 100 \mu \mathrm{m}$ at the point where the radiation is produced. The bunch length would therefore be on the order of the laser pulse length, consistent with particle-in-cell code results. ${ }^{28}$

In the present experiment, 3-5 nJ-level pulses were collected within a limited 30 mrad collection angle, ${ }^{12}$ and $70-80 \mathrm{~nJ}$ when collecting radiation in the angular range 80 $300 \mathrm{mrad}$. For the latter case the charge per bunch was 0.3 nC. These measurements are consistent with predictions from theory. The measured THz pulse energy can be dramatically increased by a number of straightforward methods. Maximizing the amount of charge increases source performance, since the coherent transition radiation signal scales quadratically with bunch charge. The radiation is directed with an angular distribution which has a minimum on axis and an opening angle which is roughly $1 / \gamma$ in the absence of diffraction effects but considerably larger for plasmas with finite transverse extent $(b \sim 1) .{ }^{13}$ Increasing $\theta_{\text {coll }}$ to 200 mrad, using the present experimental setup, is expected to increase the radiated energy per pulse to $>1 \mu \mathrm{J}$ for a bunch charge greater than $1 \mathrm{nC}$. Furthermore, as shown in Eq. (6), $100 \mu \mathrm{J} /$ pulse could be achieved by increasing the transverse plasma size $\rho$ to the mm-scale, thus minimizing diffractive effects. This could be done by focusing a laser beam with cylindrical optics to produce a vertical mm-scale sheet-like plasma through which the electron beam propagates. Increasing the mean energy of the electron beam to the few 10's of $\mathrm{MeV}$ with, e.g., a channel-guided LWFA, ${ }^{29}$ could further increase the pulse energy to the few $100 \mu \mathrm{J}$ level in a $0.1 \mathrm{rad}$ cone. Such intense terahertz radiation will provide access to nonlinear regimes and highly nonequilibrium processes using $\mathrm{MV} / \mathrm{cm}$ field strengths. It may also benefit single shot two-dimensional terahertz radiation imaging. Improving the performance of laser driven accelerator will in turn be aided by the use of coherent emission of radiation at the plasmavacuum boundary as a diagnostic tool for the electron bunch, through its sensitivity to charge, energy distribution, and bunch duration.

\section{ACKNOWLEDGMENTS}

The authors acknowledge contributions of B. Marcelis, D. Auerbach, D. Bruhwiler, B. Shadwick, L. Archambault, J. Byrd, M.C. Martin, J. Corlett, D. Li, M. Dickinson, S. DiMaggio, D. Syversrud, N. Ybarrolaza, J. Wallig, D. Calais, P. Catravas, F. Sannibale, J. Singley, and D. Chemla. This work was supported by the U.S. Department of Energy under Contract No. DE-AC03-76SF0098. C.G.R. Geddes acknowledges the Hertz Foundation for support.

${ }^{1}$ E. Esarey, P. Sprangle, J. Krall, and A. Ting, IEEE Trans. Plasma Sci. 24, 252 (1996).

${ }^{2}$ A. Modena, Z. Najmudin, A. E. Dangor, C. E. Clayton, K. A. Marsh, C. Joshi, V. Malka, C. B. Darrow, C. Danson, D. Neely, and F. N. Walsh, Nature (London) 377, 606 (1995).

${ }^{3}$ A. Ting, C. I. Moore, K. Krushelnick, C. Manka, E. Esarey, P. Sprangle, R. Hubbard, H. R. Burris, and M. Baine, Phys. Plasmas 4, 1889 (1997).

${ }^{4}$ C. Gahn, G. D. Tsakiris, A. Pukhov, J. Meyer-ter-Vehn, G. Pretzler, P. Thirolf, D. Habs, and K. J. Witte, Phys. Rev. Lett. 83, 4772 (1999).

${ }^{5}$ X. Wang, M. Krishnan, N. Saleh, H. Wang, and D. Umstadter, Phys. Rev. Lett. 84, 5324 (2000).

${ }^{6}$ M. I. K. Santala, Z. Najmudin, E. L. Clark, M. Tatarakis, K. Krushelnick, A. E. Dangor, V. Malka, J. Faure, R. Allott, and R. J. Clarke, Phys. Rev. Lett. 86, 1227 (2001).

${ }^{7}$ W. P. Leemans, P. Catravas, E. Esarey, C. Geddes, C. Toth, R. Trines, C. B. Schroeder, B. A. Shadwick, J. van Tilborg, and J. Faure, Phys. Rev. Lett. 89, 174802 (2002)

${ }^{8}$ V. Malka, S. Fritzler, E. Lefebvre, M. M. Aleonard, F. Burgy, J. P. Chambaret, J. F. Chemin, K. Krushelnick, G. Malka, S. P. D. Mangles, Z. Najmudin, M. Pittman, J. P. Rousseau, J. N. Scheurer, B. Walton, and A. E. Dangor, Science 298, 1596 (2002).

${ }^{9} \mathrm{~K}$. Krushelnick, 2003, private communication.

${ }^{10}$ W. P. Leemans, D. Rodgers, P. Catravas, C. Geddes, G. Fubiani, E. Esarey, B. Shadwick, R. Donahue, and A. Smith, Phys. Plasmas 8, 2510 (2001).

${ }^{11}$ M. I. K. Santala, M. Zepf, F. N. Beg, E. L. Clark, A. E. Dangor, K. Krushelnick, M. Tatarakis, I. Watts, K. W. D. Ledingham, T. McCanny, I. Spencer, A. C. Machacek, R. Allott, R. Clarke, and P. A. Norreys, Appl. Phys. Lett. 78, 19 (2001).

${ }^{12}$ W. P. Leemans, C. Geddes, J. Faure, C. Tóth, J. van Tilborg, C. B. Schroeder, E. Esarey, G. Fubiani, D. Auerbach, B. Marcelis, M. Carnahan, R. Kaindl, J. Byrd, and M. Martin, Phys. Rev. Lett. 91, 074802 (2003). 
${ }^{13}$ C. B. Schroeder, E. Esarey, J. van Tilborg, and W. P. Leemans, Phys. Rev. E (in preparation).

${ }^{14}$ A. S. Weling, B. B. Hu, N. M. Froberg, and D. H. Auston, Appl. Phys. Lett. 64, 137 (1994).

${ }^{15}$ X.-C. Zhang, B. B. Hu, J. T. Darrow, and D. H. Auston, Appl. Phys. Lett. 56, 1011 (1990).

${ }^{16}$ E. Budiarto, J. Margolies, S. Jeong, J. Son, and J. Bokor, IEEE J. Quantum Electron. 32, 1839 (1996).

${ }^{17}$ M. L. Ter-Mikaelian, High-energy electromagnetic processes in condensed media (Wiley-Interscience, New York, 1972).

${ }^{18}$ U. Happek, A. J. Sievers, and E. B. Blum, Phys. Rev. Lett. 67, 2962 (1991).

${ }^{19}$ Y. Shibata, T. Takahashi, T. Kanai, K. Ishi, M. Ikezawa, J. Ohkuma, S. Okuda, and T. Okada, Phys. Rev. E 50, 1479 (1994).

${ }^{20}$ G. Dugan, A. Misuri, and W. Leemans, Design and performance estimates for the l'oasis experiment magnetic spectrometers, Technical Report LBNL-49394, Lawrence Berkeley National Laboratory, University of California, Berkeley, CA 94720, 2001.

${ }^{21}$ C. B. Schroeder, E. Esarey, C. G. R. Geddes, C. Tóth, B. A. Shadwick, J. van Tilborg, J. Faure, and W. P. Leemans, Phys. Plasmas 10, 2039 (2003).
${ }^{22}$ T. Hosokai, K. Kinoshita, A. Zhidkov, K. Nakamura, T. Watanabe, T. Ueda, H. Kotaki, M. Kando, K. Nakajima, and M. Uesaka, Phys. Rev. E 67, 036407 (2003).

${ }^{23}$ S.-Y. Chen, M. Krishnan, A. Maksimchuk, R. Wagner, and D. Umstadter, Phys. Plasmas 6, 4739 (1999).

${ }^{24}$ A. W. Chao, R. Pitthan, T. Tajima, and D. Yeremian, Phys. Rev. ST Accel. Beams 6, 024201 (2003).

${ }^{25}$ G. Fubiani, G. Dugan, W. Leemans, E. Esarey, and J. L. Bobin, Semianalytical $6 \mathrm{~d}$ space charge model for dense electron bunches with large energy spreads, in Proc. of the 2002 Advanced Accelerator Concepts Workshop, edited by C. E. Clayton and P. Muggli, Vol. 647, pp. 203-212, (American Institute of Physics, Melville NY, 2002).

${ }^{26}$ S. Fritzler, 2003, private communication.

${ }^{27}$ D. Grischkowsky, S. Keiding, M. van Exter, and C. Fattinger, J. Opt. Soc. Am. B 7, 2006 (1990).

${ }^{28}$ K. C. Tzeng, W. B. Mori, and T. Katsouleas, Phys. Rev. Lett. 79, 5258 (1997).

${ }^{29}$ A. J. W. Reitsma, W. P. Leemans, E. Esarey, C. B. Schroeder, L. P. J. Kamp, and T. J. Schep, Phys. Rev. ST Accel. Beams 5, 051301 (2002). 